\title{
On the Associative Nijenhuis Relation
}

\author{
Kurusch Ebrahimi-Fard *
}

\author{
Institut Henri Poincaré \\ 11, rue Pierre et Marie Curie \\ F-75231 Paris Cedex 05 \\ FRANCE \\ kurusch@ihes.fr \\ and
}

Universität Bonn - Physikalisches Institut

Nussallee 12, D-53115 Bonn

Germany

fard@th.physik.uni-bonn.de

Submitted: Mar 22, 2004; Accepted: Jun 1, 2004; Published: Jun 16, 2004

MR Subject Classifications: 08B20, 83C47, 35Q15, 17A30

\begin{abstract}
We give the construction of a free commutative unital associative Nijenhuis algebra on a commutative unital associative algebra based on an augmented modified quasi-shuffle product.
\end{abstract}

Keywords: quasi-shuffle, modified quasi-shuffle, associative Nijenhuis relation, Rota-Baxter relation, Loday-type algebras.

\section{Introduction}

The associative analog of the Nijenhuis relation [6] may be regarded as the homogeneous version of the Rota-Baxter relation $[31,29,28,30,3,4,7,9]$. Some of its algebraic aspects especially

${ }^{*}$ On leave from Bonn University, Phys. Inst., Theory Dept. This work was done when the author was visiting the Center for Mathematical Physics at Boston University, 111 Cummington Street, Boston, MA 02215, USA. 
with regard to the notion of quantum bihamiltonian systems were investigated by Carinena et al. in [6]. The Lie algebraic version of the associative Nijenhuis relation is investigated in $[12,13]$ in the context of the classical Yang-Baxter equation, which is closely related to the Lie algebraic version of the Rota-Baxter relation (see especially [5]). Likewise in [18] the deformations of Lie brackets defined by Nijenhuis operators and in [17] the connections to the (modified) classical Yang-Baxter relation are studied. In Kreimer's work $[19,20]$ the connection of the Rota-Baxter relation to the Riemann-Hilbert problem in the realm of perturbative Quantum Field Theory is reviewed.

The algebraic properties of the associative notion of the Nijenhuis relation, respectively $\mathrm{Ni}$ jenhuis algebras, provide interesting insights into associative analogs of Lie algebraic structures. Also, both the Rota-Baxter and the associative Nijenhuis relation are of interest with respect to the Hopf algebraic formulation of the theory of renormalization in perturbative Quantum Field Theory and especially to aspects of integrable systems.

After defining a commutative unital associative Nijenhuis algebra we use an augmented modified quasi-shuffle product to give explicitly the construction of the free commutative unital associative Nijenhuis algebra generated by a commutative unital associative $\mathbb{K}$-algebra. We follow thereby closely the inspiring work of Guo et al. $[14,15]$. The main aspect of this construction of the free object is the use of a "homogenized" notion of Hoffman's quasi-shuffle product [16] giving an associative, unital, and commutative composition. This natural ansatz relies on the close resemblance between the Rota-Baxter and the associative Nijenhuis relation. The free construction of the former is essentially given by a modified quasi-shuffle product, called mixable shuffle in [14].

Let us remark here that in recent papers $[9,1,2]$ it was shown that non-commutative ${ }^{1}$ Rota- $^{-}$ Baxter algebras always give Loday-type algebras, i.e. dendriform di- and trialgebra structures $[22,23,24]$.

Also, we would like to point the reader to recent interesting work, which appeared after this article was prepared. In [10,21], the associative Nijenhuis identity and its related shuffle relation were linked to Loday-type structures in a detailed manner, giving rise to new structures in this realm.

The paper is organized as follows. In Section 2 we give the definition of a commutative, unital and associative Nijenhuis $\mathbb{K}$-algebra and the associative Nijenhuis relation. We introduce the notion of Nijenhuis homomorphism and free Nijenhuis algebra. In Section 3 we define the modified and augmented modified quasi-shuffle product and establish their properties. In Section 4 the augmented modified quasi-shuffle product algebra is identified as the free commutative associative unital Nijenhuis algebra, thereby giving an explicit construction of the latter. This section closes with a remark concerning the connection to Loday-type algebras.

Throughout this paper, we will consider $\mathbb{K}$ to be a commutative field of characteristic zero. The term $\mathbb{K}$-algebra always means if not stated otherwise associative commutative unital $\mathbb{K}$ algebra.

\footnotetext{
${ }^{1}$ not necessarily commutative
} 


\section{The associative Nijenhuis Relation}

Definition 2.1. A Nijenhuis $\mathbb{K}$-algebra is a pair $(\mathcal{A}, N)$, where $\mathcal{A}$ is a $\mathbb{K}$-algebra and $N: \mathcal{A} \rightarrow \mathcal{A}$ is a linear operator satisfying the associative Nijenhuis relation:

$$
N(x) N(y)+N^{2}(x y)=N(N(x) y+x N(y)), \quad x, y \in \mathcal{A} .
$$

The map $N: \mathcal{A} \rightarrow \mathcal{A}$ might be called associative Nijenhuis operator or just Nijenhuis map for short.

Remark 2.2. (i) In this setting "associative" refers to the relation (1) to distinguish it clearly from its well-known Lie algebraic version [6, 12, 13, 18, 17]:

$$
[N(x), N(y)]+N^{2}([x, y])=N([N(x), y]+[x, N(y)]) .
$$

(ii) A Nijenhuis map on a $\mathbb{K}$-algebra $\mathcal{A}$ gives also a Nijenhuis map for the associated Lie algebra $(\mathcal{A},[]$,$) , where [$,$] is the commutator.$

(iii) With $N: \mathcal{A} \rightarrow \mathcal{A}$ being a Nijenhuis operator, also the operator $\widehat{N}:=i d_{\mathcal{A}}-N$ satisfies relation (1).

Equation (1) may be interpreted as the homogeneous version of the standard form of the Rota-Baxter relation of weight $\lambda \in \mathbb{K}[9]$ :

$$
R(x) R(y)+\lambda R(x y)=R(R(x) y+x R(y)) .
$$

With respect to the Rota-Baxter relation, a simple transformation $R \rightarrow \lambda^{-1} R$ gives the so called standard form of (3), i.e., weight $\lambda=1$. The homogeneity of relation (1) destroys this freedom to renormalize the operator $N$, so as to allow for either sign in front of the second term on the left-hand side of (1) for instance.

Let us give two examples of operators fulfilling the associative Nijenhuis relation.

Example 2.3. (i) Left or right multiplication $L_{a} b:=a b, R_{a} b:=b a, a, b \in \mathcal{A}$ both satisfy relation (1).

(ii) Another class of associative Nijenhuis operators comes from idempotent Rota-Baxter operators. Let $(\mathcal{A}, R)$ be a Rota-Baxter algebra with $R$ being an idempotent $\left(R^{2}=R\right)$ Rota-Baxter operator satisfying relation (3) for $\lambda=1$. The operator $\widehat{R}:=i d_{\mathcal{A}}-R$ being idempotent, too, also satisfies equation (3) (for $\lambda=1$ ). Define the following operator:

$$
N_{\tau}:=\widehat{R}-\tau R=i d_{\mathcal{A}}-(\tau+1) R, \quad \tau \in \mathbb{K} .
$$

The map $N_{\tau}$ is an associative Nijenhuis operator. The case $\tau=1$ relates to the interesting modified Rota-Baxter relation:

$$
N_{1}(x) N_{1}(y)+x y=N_{1}\left(N_{1}(x) y+x N_{1}(y)\right)
$$

One finds similar examples and further algebraic aspects related to equation (1) in [6]. 


\subsection{Nijenhuis Homomorphism and the Universal Property}

We introduce now the notion of a Nijenhuis and Rota-Baxter algebra homomorphism.

Definition 2.4. We call an algebra homomorphism $\phi: \mathcal{A}_{1} \rightarrow \mathcal{A}_{2}$ between Nijenhuis (respectively Rota-Baxter) $\mathbb{K}$-algebras $\mathcal{A}_{1}, \mathcal{A}_{2}$ a Nijenhuis (resp. Rota-Baxter) homomorphism if and only if $\phi$ intertwines with the Nijenhuis (resp. Rota-Baxter) operators $N_{1}, N_{2}$ (resp. $\left.R_{1}, R_{2}\right)$ :

$$
\phi \circ X_{1}=X_{2} \circ \phi, \quad X_{i}=N_{i}\left(\text { resp. } R_{i}\right), i=1,2 .
$$

Definition 2.5. A Nijenhuis (respectively Rota-Baxter) $\mathbb{K}$-algebra $F(\mathcal{A})$ generated by a $\mathbb{K}$ algebra $\mathcal{A}$ together with an associative algebra homomorphism $j_{\mathcal{A}}: \mathcal{A} \rightarrow F(\mathcal{A})$ is called a free Nijenhuis (resp. Rota-Baxter of weight $\lambda \in \mathbb{K}$ ) $\mathbb{K}$-algebra if the following universal property is satisfied: for any associative Nijenhuis (resp. Rota-Baxter) $\mathbb{K}$-algebra $\mathcal{X}$ and $\mathbb{K}$-algebra homomorphism $\phi: \mathcal{A} \rightarrow \mathcal{X}$ there exists a unique Nijenhuis (resp. Rota-Baxter) homomorphism $\tilde{\phi}: F(\mathcal{A}) \rightarrow X$ such that the diagram:

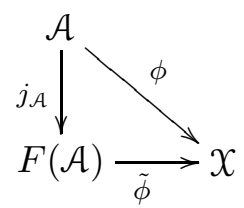

commutes.

In the next section we introduce Hoffman's quasi-shuffle product and an augmented respectively augmented modified version of it to give the construction of the free Nijenhuis algebra.

\section{Modified Quasi-Shuffle Product}

Let $\mathcal{A}$ be a $\mathbb{K}$-algebra. We denote the product on $\mathcal{A}$ by $[a b] \in \mathcal{A}, a, b \in \mathcal{A}$ and the algebra unit by $e \in \mathcal{A},[e a]=a, a \in \mathcal{A}$.

Consider the tensor module of $\mathcal{A}$ :

$$
\mathcal{T}(\mathcal{A}):=\bigoplus_{n \geq 0} \mathcal{A}^{\otimes^{n}}
$$

where $\mathcal{A}^{\otimes^{0}}=\mathbb{K}$. We denote generators $a_{1} \otimes \cdots \otimes a_{n}$ by concatenation i.e., words $a_{1} \ldots a_{n}$. Generally we use capitals $U \in \mathcal{A}^{\otimes^{n}}, n>1$ for words and lower case letters $a \in \mathcal{A}$ for letters. The natural grading on $\mathcal{T}(\mathcal{A})$ is defined by the length $\mathfrak{l}$ of words, such that $\mathfrak{l}(U V):=\mathfrak{l}(U)+\mathfrak{l}(V)=$ $n+m$, for $U \in \mathcal{A}^{\otimes^{n}}$ and $V \in \mathcal{A}^{\otimes^{m}}, n, m \geq 0$ i.e., for the empty word denoted by $1 \in \mathbb{K}=\mathcal{A}^{\otimes^{0}}$ we have $\mathfrak{l}(1)=0$. We denote the associative commutative quasi-shuffle product $[16]$ on $\mathcal{T}(\mathcal{A})$ by:

$$
a U * b V:=a(U * b V)+b(a U * V)-\lambda[a b](U * V)
$$

$\lambda \in \mathbb{K}$ being an arbitrary parameter. The "merged" term $[a b] \in \mathcal{A}$ gives a letter. The empty word $1 \in \mathcal{T}(\mathcal{A})$ gives the product unit, i.e. $1 * U:=U * 1:=U, U \in \mathcal{T}(\mathcal{A})$. 
Remark 3.1. (1) If $\lambda=0$ we get the ordinary shuffle product of two words $U:=u_{1} \cdots u_{n}, V=$ $v_{1} \cdots v_{m} \in \mathcal{T}(\mathcal{A})[16]$, denoted in general by:

$$
U \sqcup \sqcup V:=u_{1}\left(u_{2} \cdots u_{n} \sqcup v_{1} \cdots v_{m}\right)+v_{1}\left(u_{1} \cdots u_{n} \sqcup \sqcup v_{2} \cdots v_{m}\right) .
$$

The shuffle sum in (9) consists of $\left(\begin{array}{c}m+n \\ m\end{array}\right)$ words.

(2) With $\lambda \in \mathbb{K}$ it is obvious that the quasi-shuffle product $*$ is not grade preserving with respect to the length $\mathfrak{l}$ of words, due to the last term on the right-hand side of (8).

Let us mention here, that Hoffman assumes the algebra $\mathcal{A}$ to be equipped with a grading deg $g_{\mathcal{A}}$ which is preserved by the product []$\in \mathcal{A}$ i.e., $\operatorname{deg}_{\mathcal{A}}([a b])=\operatorname{deg}_{\mathcal{A}}(a)+\operatorname{deg}_{\mathcal{A}}(b)$. This grading is extended to $\mathcal{T}(\mathcal{A})$ by identifying the degree of a word $a_{1} \ldots a_{n}$ with the sum of the degrees of the letters $a_{1}, \cdots, a_{n}$. The above $*$-product then becomes grade preserving with respect to this second grading on $\mathcal{T}(\mathcal{A})$. We refer the reader to [16] and [11] for details, related to Hopf algebraic aspects related to the quasi-shuffle product construction.

Here we will work exclusively with the natural grading $\mathfrak{l}$ given by the length of words.

We define now the following two linear operators on $\mathcal{T}(\mathcal{A})$ :

Definition 3.2. Let $B_{a}^{+}, B^{-}: \mathcal{T}(\mathcal{A}) \rightarrow \mathcal{T}(\mathcal{A}), a \in \mathcal{A}$ be the linear operators with:

$$
\begin{aligned}
B_{a}^{+}\left(a_{1} \ldots a_{n}\right) & :=a a_{1} \ldots a_{n} \\
B^{-}\left(a_{1} \ldots a_{n}\right) & :=a_{2} \ldots a_{n} .
\end{aligned}
$$

Proposition 3.3. Denote the set of idempotent elements in $\mathcal{A}$ by $\mathcal{J}:=\{x \in \mathcal{A} \mid[x x]=x\}$. The linear operator $B_{x}^{+}$on $(\mathcal{T}(\mathcal{A}), *)$ satisfies the Rota-Baxter relation (3) of weight $\lambda \in \mathbb{K}$ when $x \in \mathcal{J}$.

Proof. This follows by construction of the quasi-shuffle product i.e., relation (8).

Remark 3.4. For $\lambda=0$ i.e., the ordinary shuffle relation, $B_{x}^{+}$is a Rota-Baxter map of zero weight for general $x \in \mathcal{A}$.

The augmented quasi-shuffle product on the augmented tensor module:

$$
\overline{\mathcal{T}}(\mathcal{A}):=\bigoplus_{n>0} \mathcal{A}^{\otimes^{n}}
$$

is defined in the following way:

$$
a U \triangleright b V:=[a b](U * V)
$$

whereby the unit of this product is given by the algebra unit $e \in \mathcal{A}, e \boxminus U=U \boxminus e=U$, $U \in \overline{\mathcal{T}}(\mathcal{A})$. The associativity and commutativity of $\square$ for arbitrary $\lambda \in \mathbb{K}$ follows from the associativity and commutativity of the quasi-shuffle product and the product [ ] in $\mathcal{A}$.

Remark 3.5. The operator $B_{x}^{+}$on $(\overline{\mathcal{T}}(\mathcal{A}), \square)$ is Rota-Baxter of weight $\lambda \in \mathbb{K}$ only if $x$ is the unit $e \in \mathcal{A}$.

Definition 3.6. We define now a modified quasi-shuffle product on $\mathcal{T}(\mathcal{A})$ using the algebra unit $e \in \mathcal{A}$ :

$$
a U \circledast b V:=a(U \circledast b V)+b(a U \circledast V)-\lambda e[a b](U \circledast V) .
$$

For the empty word we have $1 \circledast U=U \circledast 1:=U, U \in \mathcal{T}(\mathcal{A})$. 
Remark 3.7. Commutativity of $\circledast$ on $\mathcal{T}(\mathcal{A})$ follows from commutativity of the product [] in $\mathcal{A}$.

Lemma 3.8. The bilinear product $\circledast$ on $\mathcal{T}(\mathcal{A})$ defined above is associative if and only if $\lambda=1$ in (14).

Proof. We prove the associativity property for this product by induction on the length of words. The linearity allows us to reduce the proof to generators in $\mathcal{T}(\mathcal{A})$. Choose $a, b, c \in \mathcal{T}(\mathcal{A})$, $\mathfrak{l}(a)=\mathfrak{l}(b)=\mathfrak{l}(c)=1$ :

$$
\begin{aligned}
a \circledast(b \circledast c)= & a \circledast(b c+c b-\lambda e[b c]) \\
= & a b c+b(a c+c a-\lambda e[a c])-\lambda e[a b] c \\
& \quad+a c b+c(a b+b a-\lambda e[a b])-\lambda e[a c] b \\
& \quad-\lambda(a e[b c]+e(a[b c]+[b c] a-\lambda e[a[b c]]))+\lambda^{2}(e[a e][b c]) \\
= & a b c+b a c+b c a+a c b+c a b+c b a \\
& -\lambda(b e[a c]+e[a b] c+c e[a b]+e[a c] b+a e[b c]+e a[b c]+e[b c] a) \\
& \quad+\lambda^{2}(e e[a[b c]]+e a[b c]) \\
(a \circledast b) \circledast c= & (a b+b a-\lambda e[a b]) \circledast c \\
= & a(b c+c b-\lambda e[b c])+c a b-\lambda e[a c] b \\
& \quad+b(a c+c a-\lambda e[a c])+c b a-\lambda e[b c] a \\
& \quad-\lambda(e([a b] c+c[a b]-\lambda e[[a b] c])+c e[a b])+\lambda^{2}(e[e c][a b]) \\
= & a b c+a c b+c a b+b a c+b c a+c b a \\
& -\lambda(a e[b c]+e[a c] b+b e[a c]+e[b c] a+e[a b] c+e c[a b]+c e[a b]) \\
& \quad+\lambda^{2}(e e[[a b] c]+e c[a b])
\end{aligned}
$$

We see already here that this product is associative if and only if $\lambda=1$, eliminating the two unwanted terms in the $\lambda^{2}$-part, i.e. $e c[a b]$ and $e a[b c]$ ! Now we assume that associativity is true for $\mathfrak{l}(U)+\mathfrak{l}(V)+\mathfrak{l}(W)=k-1>3$ and choose $a X, b Y, c Z \in \mathfrak{T}(\mathcal{A}), \mathfrak{l}(a X)+\mathfrak{l}(b Y)+\mathfrak{l}(c Z)=k$ (we now use $\lambda=1$ ):

$$
\begin{aligned}
a X \circledast(b Y \circledast c Z)= & a X \circledast(b(Y \circledast c Z)+c(b Y \circledast Z)-e[b c](Y \circledast Z)) \\
= & a X \circledast b(Y \circledast c Z)+a X \circledast c(b Y \circledast Z)-a X \circledast e[b c](Y \circledast Z) \\
= & a(X \circledast b(Y \circledast c Z))+b(a X \circledast(Y \circledast c Z))-e[a b](X \circledast(Y \circledast c Z)) \\
& +a(X \circledast c(b Y \circledast Z))+c(a X \circledast(b Y \circledast Z))-e[a c](X \circledast(b Y \circledast Z)) \\
& -a(X \circledast e[b c](Y \circledast Z))+e(a X \circledast[b c](Y \circledast Z))+e[a e](X \circledast[b c](Y \circledast Z)) \\
= & a((X \circledast b Y) \circledast c Z)+b((a X \circledast Y) \circledast c Z)-c((a X \circledast b Y) \circledast Z) \\
& +e e[[a b] c]((X \circledast Y) \circledast Z)-e[a c]((X \circledast b Y) \circledast Z) \\
& -e[b c]((a X \circledast Y) \circledast Z)-e[a b]((X \circledast Y) \circledast c Z) \\
= & (a X \circledast b Y) \circledast c Z
\end{aligned}
$$

The modified quasi-shuffle is homogeneous with respect to the length of the words in contrast to the ordinary quasi-shuffle product (8). Anticipating the result in the next section we mention here that this fact reflects the homogeneity difference between the Rota-Baxter relation (3) and the associative Nijenhuis relation (1). 
Lemma 3.9. For the algebra $(\mathcal{T}(\mathcal{A}), \circledast)$, the operator $B_{e}^{+}$satisfies the associative Nijenhuis relation only if $x$ is the unit $e \in \mathcal{A}$ :

$$
B_{e}^{+}(X) \circledast B_{e}^{+}(Y)=B_{e}^{+}\left(X \circledast B_{e}^{+}(Y)\right)+B_{e}^{+}\left(B_{e}^{+}(X) \circledast Y\right)-B_{e}^{+} B_{e}^{+}(X \circledast Y) .
$$

Proof. Again, this result follows from the construction of the modified quasi-shuffle product.

Proposition 3.10. Let the augmented modified quasi-shuffle product on the augmented tensor module $\overline{\mathcal{T}}(\mathcal{A})$ be defined as follows for $a, b \in \mathcal{A}$ and $U, V \in \overline{\mathcal{T}}(\mathcal{A})$ :

$$
a U \otimes b V:=[a b](U \circledast V) .
$$

Then $\otimes$ is associative and commutative, and we have $e \otimes V=V \otimes e=V$, for $V \in \overline{\mathcal{T}}(\mathcal{A})$ and the unit $e \in \mathcal{A}$.

In the following section we will show that the triple $\left(\overline{\mathcal{T}}(\mathcal{A}), \otimes, B_{e}^{+}\right)$defines a Nijenhuis algebra; moreover, we will see that it fulfills the universal property.

\section{Free Associative Nijenhuis Algebra}

We will use the augmented modified quasi-shuffle product $\Delta$ to give the construction of the free Nijenhuis $\mathbb{K}$-algebra. The existence of such an object follows from general arguments in the theory of universal algebras $[30,8]$ since the category of Nijenhuis $\mathbb{K}$-algebras, defined through the identity (1) forms a variety (in the sense of universal algebras, see especially section 6 of [30] for a concise summary of the main ideas).

The following construction of the free associative Nijenhuis algebra $(\overline{\mathcal{T}}(\mathcal{A}), \otimes)$ was inspired by the mixable shuffle product of Guo et al. [14].

Let us remark here that the construction of the free Rota-Baxter algebra (of weight $\lambda \neq 0$ ) uses the augmented quasi-shuffle product $\square$, defined in (13) and works analogously to what follows.

The quasi-shuffle product $*$ essentially embodies the structure of the Rota-Baxter relation (3). The case of weight $\lambda=0$ gives the "trivial" Rota-Baxter algebra, i.e. relation (3) without the second term on the left-hand side. This construction was essentially given in [14] using a non-recursive notion of a so called mixable shuffle product. However the formulation using Hoffman's quasi-shuffle product [16] is new. For a detailed account of the link between the mixable and quasi-shuffle product, also addressing Hopf algebraic aspects, we refer the reader to the results in the recent work [11].

The essential point (for both constructions of the free objects) lies in the (augmented) quasishuffle product (or mixable shuffle in [14]) and its "merging" of letters $\lambda[a b], a, b \in \mathcal{A}$ in the *-product on the right-hand side of (13), whereby $\lambda \in \mathbb{K}$ is arbitrary. It represents the second term on the left-hand side of (3). The modified quasi-shuffle product $\circledast$ (and hence the augmented modified quasi-shuffle product $\otimes)$ reflects relation (1): in particular, the third term on the righthand side of (14) is related to the second term on the left-hand side of the associative Nijenhuis relation (1). It has to be underlined that the associativity of $\square$ relies on the choice $\lambda=1$ in equation (14). This is due to the fact [6] that the following bilinear map on a $\mathbb{K}$-algebra $\mathcal{A}, N$ being an arbitrary $\mathbb{K}$-linear map:

$$
\mu_{N}(a, b):=N(a) b+a N(b)-N(a b)
$$


gives an associative product if and only if the following map, called $\mu$-Nijenhuis torsion in [6]:

$$
T_{\mu, N}(a, b):=N\left(\mu_{N}(a, b)\right)-N(a) N(b)
$$

is a 2-Hochschild cocycle. Of course this holds if $N$ is a Nijenhuis operator, since relation (1) implies that the right-hand side of (18) is zero.

In the sequel we will prove the following theorem.

Theorem 4.1. For a commutative, associative, unital $\mathbb{K}$-algebra $\mathcal{A},\left(\overline{\mathcal{T}}(\mathcal{A}), \bigotimes, B_{e}^{+}\right)$is the free (associative) Nijenhuis algebra generated by $\mathcal{A}$.

Proof. The proof of the above Theorem (4.1) will be divided into three parts. In the following Proposition (4.2), we show that $\left(\overline{\mathcal{T}}(\mathcal{A}), \bigotimes, B_{e}^{+}\right)$is a Nijenhuis algebra i.e., the operator $B_{e}^{+}$ satisfies the associative Nijenhuis relation.

Proposition 4.2. $\overline{\mathcal{T}}(\mathcal{A})$ together with the augmented modified quasi-shuffle product $\otimes$ and the linear map $B_{e}^{+}$is an associative Nijenhuis algebra.

Proof.

$$
\begin{aligned}
B_{e}^{+}(U) \otimes B_{e}^{+}(V)+\left(B_{e}^{+}\right)^{2}(U \otimes V)= & e U \otimes e V+e e(U \otimes V) \\
= & {[e e](U \circledast V)+e e\left(\left[u_{1} v_{1}\right]\left(B^{-}(U) \circledast B^{-}(V)\right)\right) } \\
= & e\left(u_{1}\left(B^{-}(U) \circledast V\right)+v_{1}\left(U \circledast B^{-}(V)\right)\right. \\
& \left.-e\left[u_{1} v_{1}\right]\left(B^{-}(U) \circledast B^{-}(V)\right)\right) \\
& +e e\left[u_{1} v_{1}\right]\left(B^{-}(U) \circledast B^{-}(V)\right) \\
= & B_{e}^{+}\left(U \otimes B_{e}^{+}(V)+B_{e}^{+}(U) \otimes V\right)
\end{aligned}
$$

For the triple $\left(\overline{\mathcal{T}}(\mathcal{A}), \otimes, B_{e}^{+}\right)$to be the free Nijenhuis algebra over $\mathcal{A}$ we have to show that the universal property is satisfied. Let $\mathcal{U}$ be an arbitrary Nijenhuis $\mathbb{K}$-algebra with Nijenhuis operator $N$ and $\phi: \mathcal{A} \rightarrow \mathcal{U}$ an $\mathbb{K}$-algebra homomorphism.

We have to extend the $\mathbb{K}$-algebra map $\phi$ to a Nijenhuis homomorphism $(6) \tilde{\phi}: \overline{\mathcal{T}}(\mathcal{A}) \rightarrow \mathcal{U}$ using the following important fact:

Lemma 4.3. Every generator $a_{1} \ldots a_{n} \in \overline{\mathcal{T}}(\mathcal{A})$ can be written using the modified augmented quasi-shuffle product:

$$
a_{1} \ldots a_{n}=a_{1} \otimes B_{e}^{+}\left(a_{2} \otimes B_{e}^{+}\left(a_{3} \otimes B_{e}^{+}\left(\ldots B_{e}^{+}\left(a_{n-1} \otimes B_{e}^{+}\left(a_{n}\right)\right) \ldots\right)\right)\right) .
$$

Since the extension $\tilde{\phi}$ is supposed to be a Nijenhuis algebra homomorphism we have the unique possible extension:

$$
\tilde{\phi}\left(a_{1} \ldots a_{n}\right):=\phi\left(a_{1}\right) N\left(\phi\left(a_{2}\right) N\left(\ldots N\left(\phi\left(a_{n-1}\right) N\left(\phi\left(a_{n}\right)\right)\right) \ldots\right)\right) .
$$

Defining the map $N_{w}: \mathcal{U} \rightarrow \mathcal{U}, w \in \mathcal{U}$, by $N_{w}(x):=N(w x), x \in \mathcal{U}$, we can write (20) in terms of iterated compositions (cf. $\S 4$ of [14]):

$$
\tilde{\phi}\left(a_{1} \ldots a_{n}\right)=\phi\left(a_{1}\right)\left\{\circ_{i=2}^{n} N_{\phi\left(a_{i}\right)}\left(1_{u}\right)\right\} .
$$

To complete the proof of Theorem (4.1) it remains to show the following: 
Proposition 4.4. $\tilde{\phi}$ is a Nijenhuis homomorphism.

Proof. By construction $\tilde{\phi}$ is a well defined $\mathbb{K}$-linear map. The Nijenhuis property (6) follows as in $[17, \mathrm{p} .140]$ :

$$
\begin{aligned}
& \tilde{\phi}\left(B_{e}^{+}\left(a_{1} \ldots a_{n}\right)\right)=\tilde{\phi}\left(e a_{1} \ldots a_{n}\right) \\
& \stackrel{(20)}{=} \circ_{i=1}^{n} N_{\phi\left(a_{i}\right)}\left(1_{\mathcal{U}}\right) \\
& =N\left(\phi\left(a_{1}\right) \circ_{i=2}^{n} N_{\phi\left(a_{i}\right)}\left(1_{u}\right)\right) \\
& =N\left(\tilde{\phi}\left(a_{1} \ldots a_{n}\right)\right) \text {. }
\end{aligned}
$$

Finally we would like to show that $\tilde{\phi}$ is a $\mathbb{K}$-algebra homomorphism, i.e. preserves multiplication. This we proof by induction on the length of words, $\mathfrak{l}(U)+\mathfrak{l}(V)=m+n=k$. For $k=2$ we have, $a, b \in \overline{\mathcal{T}}(\mathcal{A}):$

$$
\begin{aligned}
\tilde{\phi}(a \otimes b) & =\tilde{\phi}([a b]) \\
& \stackrel{(20)}{=} \phi([a b]) \\
= & \phi(a) \phi(b)=\tilde{\phi}(a) \tilde{\phi}(b) .
\end{aligned}
$$

Let $U:=u_{1} \ldots u_{n} \in \mathcal{A}^{\otimes^{n}}, V:=v_{1} \ldots v_{m} \in \mathcal{A}^{\otimes^{m}}$, with $m+n>2$ :

$$
\begin{aligned}
& \tilde{\phi}(U \otimes V)=\tilde{\phi}\left(\left[u_{1} v_{1}\right]\left\{B^{-}(U) \circledast B^{-}(V)\right\}\right) \\
& =\tilde{\phi}\left([ u _ { 1 } v _ { 1 } ] \left\{B_{e}^{+}\left(B^{-}(U)\right) \otimes B^{-}(V)+B^{-}(U) \otimes B_{e}^{+}\left(B^{-}(V)\right)\right.\right. \\
& \left.\left.-B_{e}^{+}\left(B^{-}(U) \otimes B^{-}(V)\right)\right\}\right) \\
& \stackrel{(20)}{=} \phi\left(u_{1}\right) \phi\left(v_{1}\right) N\left(\tilde { \phi } \left\{B_{e}^{+}\left(B^{-}(U)\right) \otimes B^{-}(V)\right.\right. \\
& \left.\left.+B^{-}(U) \otimes B_{e}^{+}\left(B^{-}(V)\right)-B_{e}^{+}\left(B^{-}(U) \otimes B^{-}(V)\right)\right\}\right) \\
& =\phi\left(u_{1}\right) \phi\left(v_{1}\right) N\left(\tilde{\phi}\left(B_{e}^{+}\left(B^{-}(U)\right)\right) \tilde{\phi}\left(B^{-}(V)\right)\right. \\
& \left.+\tilde{\phi}\left(B^{-}(U)\right) \tilde{\phi}\left(B_{e}^{+}\left(B^{-}(V)\right)\right)-N\left\{\tilde{\phi}\left(B^{-}(U)\right) \tilde{\phi}\left(B^{-}(V)\right)\right\}\right) \\
& =\phi\left(u_{1}\right) \phi\left(v_{1}\right) N\left\{N\left(\tilde{\phi}\left(B^{-}(U)\right)\right) \tilde{\phi}\left(B^{-}(V)\right)\right. \\
& +\tilde{\phi}\left(B^{-}(U)\right) N\left(\tilde{\phi}\left(B^{-}(V)\right)\right)-N\left(\tilde{\phi}\left(B^{-}(U)\right) \tilde{\phi}\left(B^{-}(V)\right)\right\} \\
& \stackrel{(\#)}{=} \quad \phi\left(u_{1}\right) \phi\left(v_{1}\right) N\left\{\tilde{\phi}\left(B^{-}(U)\right)\right\} N\left\{\tilde{\phi}\left(B^{-}(V)\right)\right\} \\
& =\phi\left(u_{1}\right) N\left\{\tilde{\phi}\left(B^{-}(U)\right)\right\} \phi\left(v_{1}\right) N\left\{\tilde{\phi}\left(B^{-}(V)\right\}\right. \\
& =\tilde{\phi}(U) \tilde{\phi}(V)
\end{aligned}
$$

In the third line we used the Nijenhuis property (22). Apparently by going from line (\#) to the next we see that the use of the augmented modified quasi-shuffle product in the above construction of the free Nijenhuis algebra is limited to the commutative case.

This finishes the proof of Theorem (4.1), showing that $\left(\overline{\mathcal{T}}(\mathcal{A}), \otimes, B_{e}^{+}\right)$is the free Nijenhuis algebra generated by $\mathcal{A}$. 
Remark 4.5. (1) The above construction may also be used to give the free Nijenhuis algebra on a set $S$ by working with the polynomial algebra $\mathbb{K}[S]$.

(2) Using the augmented quasi-shuffle product $\square$, the proof of the universal property for the triple $\left(\overline{\mathcal{T}}(\mathcal{A}), \square, \lambda, B_{e}^{+}\right)$goes analogously, giving the free Rota-Baxter $\mathbb{K}$-algebra of weight $\lambda \in \mathbb{K}$ over $\mathcal{A}$.

As we already mentioned above, the algebras $\left(\mathcal{T}(\mathcal{A}), *, \lambda, B_{e}^{+}\right)$and $\left(\mathcal{T}(\mathcal{A}), \circledast, B_{e}^{+}\right)$define a Rota-Baxter algebra and a Nijenhuis algebra, respectively.

\subsection{Dendriform Di- and Trialgebra Structures}

We mentioned in the beginning the relation between Rota-Baxter algebras and Loday-type algebras $[9,1,2]$, i.e. dendriform di- and trialgebra structures (see $[22,23]$ for definitions and further details).

In this final part we would like to relate the above given construction of the free Rota-Baxter algebra, i.e. the augmented quasi-shuffle products to these Loday-type structures.

Let us remark again, that recently in [10] and especially in [21] further results with respect to both the associative Nijenhuis and Rota-Baxter algebras were established.

A dendriform dialgebra (respectively trialgebra) is a $\mathbb{K}$-vector space $V$ equipped with two (resp. three) binary bilinear operations $\succ$ and $\prec$ (resp. $\succ, \prec$, and $\bullet$ ), satisfying a certain set of axioms $[22,23]$, such that for $x, y \in V$ the binary composition

$$
x * y:=x \succ y+x \prec y
$$

(resp. $x * y:=x \succ y+x \prec y+x \bullet y$ ) gives an associative product, making $(V, *)$ an associative, but not necessarily commutative $\mathbb{K}$-algebra. The operations $\succ$ and $\prec$ (resp. $\succ, \prec$, and •) "split" the associative product $*[24]$.

Rota-Baxter algebras $(\mathcal{A}, R)$ (resp. associative Nijenhuis algebras), not necessarily commutative, naturally fit into this picture, as they allow to define another product on the vector space underlying the Rota-Baxter algebra $\mathcal{A}$ :

$$
x * y:=x R(y)+R(x) y-a b
$$

(resp. $x * y:=x N(y)+N(x) y-N(x y)$, see (17)), which is associative. It was shown in $[1,9]$ that this splitting defines dendrifrom di- and trialgebra structures on Rota-Baxter algebras. In [21] and later in [10] this notion was extended to associative Nijenhuis algebras.

In [26] Ronco defined a dendriform dialgebra structure on the tensor coalgebra $\overline{\mathcal{T}}(V)$ over a vector space $V$ in connection to the ordinary shuffle product (9). The two compositions $\prec, \succ$ are defined using the fact that the shuffle sum (9) of two words $X=x_{1} \ldots x_{n} Z=z_{1} \ldots z_{m}$ may be characterized by words either beginning with $x_{1}$ or $z_{1}$ :

$$
\begin{aligned}
& X \prec Z:=x_{1}\left(B^{-}(X) \sqcup \sqcup Z\right) \\
& X \succ Z:=z_{1}\left(X \sqcup \sqcup B^{-}(Z)\right) .
\end{aligned}
$$

In the context of $\overline{\mathcal{T}}(\mathcal{A})$ over the $\mathbb{K}$-algebra $\mathcal{A}$ we see how this relates to the augmented quasishuffle product $\square$, with $\lambda=0$ in the $*$-product on the right-hand side of equation (13), and therefore to the Rota-Baxter map $B_{e}^{+}$of weight 0:

$$
\begin{aligned}
& X \prec Z \quad:=X \boxminus B_{e}^{+}(Z) \\
& X \succ Z \quad:=B_{e}^{+}(X) \square Z,
\end{aligned}
$$


which gives the dendriform dialgebra structure related to the Rota-Baxter relation of weight zero found by Aguiar [1]. Assuming $\lambda \neq 0$, and using instead both Rota-Baxter maps i.e., $B_{e}^{+}$ and $\lambda i d_{\overline{\mathcal{T}}(\mathcal{A})}-B_{e}^{+}=: \widehat{B_{e}^{+}}$, we have on the free Rota-Baxter algebra $(\overline{\mathcal{T}}(\mathcal{A}), \bullet)$ over $\mathcal{A}[9]$ :

Corollary 4.6. Let $\overline{\mathcal{T}}(\mathcal{A})$ be equipped with the augmented quasi-shuffle product $\square$. The two compositions

$$
X \prec Z:=X \boxminus B_{e}^{+}(Z) \quad X \succ Z:=-\widehat{B_{e}^{+}}(X) \bullet Z, \quad X, Z \in \overline{\mathcal{T}}(\mathcal{A})
$$

defined in terms of the Rota-Baxter maps $B_{e}^{+}$and $\lambda i d_{\overline{\mathfrak{T}}(\mathcal{A})}-B_{e}^{+}=: \widehat{B_{e}^{+}}$satisfy the dendriform dialgebra relations.

Loday and Ronco related in [23] the dendriform trialgebra structure to Hoffman's quasishuffle product $*$. Here we see how this relates to the augmented quasi-shuffle product $\square$ for weight $\lambda=1$ in the $*$-product on the right-hand side of equation (13):

$$
\begin{aligned}
X \prec Z & :=x_{1}\left(B^{-}(X) * Z\right)=X \boxminus B_{e}^{+}(Z) \\
X \succ Z & :=z_{1}\left(X * B^{-}(Z)\right)=B_{e}^{+}(X) \bullet Z \\
X \bullet Z & :=\left[x_{1} z_{1}\right]\left(B^{-}(X) * B^{-}(Z)\right)=X \boxminus Z .
\end{aligned}
$$

This of course extends to general weight $\lambda \in \mathbb{K}$ i.e., the free Rota-Baxter algebra $(\overline{\mathcal{T}}(\mathcal{A}), \square)$ of weight $\lambda \neq 0$, and reduces to the case (23) for $\lambda=0$.

Let us make here an observation with respect to the work [26] of Ronco. From the previous results it follows that Rota-Baxter algebras always give dendriform algebra structures. We used in the definition of the extension of an algebra homomorphism to a Rota-Baxter homomorphism the fact, analogous to $(19)$, that the elements in $\overline{\mathcal{T}}(\mathcal{A})$ may be written using the Rota-Baxter map $B_{e}^{+}(\square$ instead of $\otimes)$ :

$$
\begin{aligned}
& a_{1} \ldots a_{n}=a_{1} \boxminus B_{e}^{+}\left(a_{2} \boxminus B_{e}^{+}\left(a_{3} \boxminus B_{e}^{+}\left(\ldots B_{e}^{+}\left(a_{n-1} \boxminus B_{e}^{+}\left(a_{n}\right)\right) \ldots\right)\right)\right) \\
& =B_{e}^{+}\left(\ldots B_{e}^{+}\left(B_{e}^{+}\left(B_{e}^{+}\left(a_{n}\right) \boxminus a_{n-1}\right) \boxminus a_{n-2}\right) \boxminus a_{n-3} \ldots\right) \bullet a_{1} \text {. }
\end{aligned}
$$

With respect to the dendriform (trialgebra) structures this may be written using either of the compositions $\prec, \succ$ and introducing the "identity" maps $\omega_{\prec, \succ}$ on $\overline{\mathcal{T}}(\mathcal{A})\left(a_{i} \in \mathcal{A}\right.$ being letters):

$$
\begin{aligned}
\omega_{\prec}\left(a_{1} \ldots a_{n}\right): & a_{1} \prec\left(a_{2} \prec\left(a_{3} \prec\left(\ldots \prec\left(a_{n-1} \prec a_{n}\right) \ldots\right)\right)\right) \\
& =\left(\left(\ldots\left(\left(a_{n} \succ a_{n-1}\right) \succ a_{n-2}\right) \succ \ldots\right) \succ a_{2}\right) \succ a_{1} \\
& =: \quad \omega_{\succ}\left(a_{n} \ldots a_{1}\right)
\end{aligned}
$$

These maps are of importance with respect to the primitive elements and brace-algebras [26, 27] in a dendriform Hopf algebra. The connection to Loday's recent work [24] has to be clarified in a future work concerning this link and the algebraic properties of the augmented modified quasi-shuffle product.

\section{Acknowledgments:}

A special vote of thanks goes to Prof. D. Kreimer for helpful comments concerning this work. Also, we would like to thank the referee for his or her passion and very helpful comments. We are grateful to Prof. Li Guo for helpful discussions. This work was supported in parts by NSF grant DMS-0205977 at the Center for Mathematical Physics at Boston University. I am also indebt to Prof. Loday for his remarks. The author is supported by the German Academic Exchange Service (DAAD). 


\section{References}

[1] M. Aguiar, Prepoisson algebras, Letters in Mathematical Physics, 54, no. 4, 2000, 263-277.

[2] M. Aguiar, Infinitesimal bialgebras, pre-Lie and dendriform algebras, Nov. 2002, preprint: ArXiv:math.QA/0211074, to appear in "Hopf Algebras: Proc. from an Int. Conf. held at DePaul University", Marcel Dekker.

[3] F. V. Atkinson, Some aspects of Baxter's functional equation, J. Math. Anal. Appl. 7, 1963, 1-30.

[4] G. Baxter, An analytic problem whose solution follows from a simple algebraic identity, Pacific J. Math. 10, 1960, 731-742.

[5] A. Belavin, V. Drinfeld, Triangle Equations and Simple Lie-Algebras, Classic Reviews in Mathematics and Mathematical Physics, 1. Harwood Academic Publishers, Amsterdam, 1998, viii+91 pp.

[6] J. Cariñena, J. Grabowski, G. Marmo, Quantum bi-Hamiltonian systems, Internat. J. Modern Phys. A, 15, no. 30, 2000, 4797-4810.

[7] P. Cartier, On the structure of free Baxter algebras, Advances in Math. 9, 1972, $253-265$.

[8] P. Cohn, Universal Algebra, Second edition. Mathematics and its Applications, 6., D. Reidel Publishing Co., Dordrecht-Boston, Mass., 1981, xv+412 pp.

[9] K. Ebrahimi-Fard, Loday-type algebras and the Rota-Baxter relation, Letters in Mathematical Physics, 61, no. 2, 2002, 139-147.

[10] K. Ebrahimi-Fard, L. Guo, On the type and type product of dendriform algebras, Feb. 2004, preprint, http://newark.rutgers.edu/ liguo/lgpapers.html

[11] K. Ebrahimi-Fard, L. Guo, Quasi-Shuffles, Mixable Shuffles and Hopf Algebras, May 2004, preprint, http://newark.rutgers.edu/ liguo/lgpapers.html

[12] I.Z. Golubchik, V.V. Sokolov, One more type of classical Yang-Baxter equation, Funct. Anal. Appl., 34, no. 4, 2000, 296-298.

[13] I.Z. Golubchik, V.V. Sokolov, Generalized Operator Yang-Baxter Equations, Integrable ODEs and Nonassociative Algebras, J. of Nonlinear Math. Phys., 7, No.2, 2000, 184-197.

[14] L. Guo, W. Keigher, Baxter algebras and shuffle products, Adv. Math., 150, no. 1, 2000, 117-149.

[15] L. Guo, Baxter algebras and differential algebras, in "Differential algebra and related topics", (Newark, NJ, 2000), 281-305, World Sci. Publishing, River Edge, NJ, 2002. 
[16] M. Hoffman, Quasi-shuffle products, J. Algebraic Combin., 11, no. 1, 2000, 49-68.

[17] Y. Kosmann-Schwarzbach, The modified Yang-Baxter equation and bi-Hamiltonian structures, Differential geometric methods in theoretical physics (Chester, 1988), 12-25, World Sci. Publishing, Teaneck, NJ, 1989.

[18] Y. Kosmann-Schwarzbach, F.Magri, Poisson-Nijenhuis structures, Ann. Inst. H. Poincar Phys. Thor., 53, no. 1, 1990, 35-81.

[19] D. Kreimer, Structures in Feynman Graphs - Hopf Algebras and Symmetries, BUCMP-02-02, Feb. 2002, preprint: ArXiv:hep-th/0202110

[20] D. Kreimer, New Mathematical Structures in Renormalizable Quantum Field Theory, Annals Phys., 303, 2003, 179-202.

[21] Ph. Leroux, Construction of Nijenhuis operators and dendriform trialgebras, Nov. 2003, preprint: ArXiv:math.QA/0311132

[22] J.-L. Loday, Dialgebras, in "Dialgebras and related operads" Springer Lecture Notes in Mathematics 1763, 2001, 7-66.

[23] J.-L. Loday, M. Ronco, Trialgebras and families of polytopes, May 2002, preprint: ArXiv:math.AT/0205043

[24] J.-L. Loday, Scindage d'associativité et algèbre de Hopf, Jan. 2003, preprint, http://www-irma.u-strasbg.fr/ loday

[25] C. Reutenauer, Free Lie Algebras, Oxford University Press, Oxford, 1993.

[26] M. Ronco, Primitive elements in a free dendriform algebra, "New trends in Hopf algebra theory" (La Falda, 1999), Contemp. Math., 267, 2000, 245-263.

[27] M. Ronco, Eulerian idempotents and Milnor-Moore theorem for certain noncommutative Hopf algebras, J. Algebra, 10, 2002, 152-172.

[28] G.-C. Rota, Baxter algebras and combinatorial identities. I, II, Bull. Amer. Math. Soc. 75, 1969, 325-329; ibid. 75, 1969, 330-334.

[29] G.-C. Rota, Baxter operators, an introduction, in "Gian-Carlo Rota on combinatorics: Introductory papers and commentaries", J. P.S. Kung Ed., Contemp. Mathematicians, Birkhäuser Boston, Boston, MA, 1995, 504-512.

[30] G.-C. Rota, D. Smith, Fluctuation theory and Baxter algebras, in "Gian-Carlo Rota on combinatorics: Introductory papers and commentaries", J. P.S. Kung Ed., Contemp. Mathematicians, Birkhäuser Boston, Boston, MA, 1995, 481-503.

[31] G.-C. Rota, Ten mathematics problems I will never solve, Mitt. Dtsch. Math.-Ver., no. 2, 1998, 45-52. 